\title{
Tabularia
}

\section{Le récit de la translation des reliques de saint Regnobert : histoire d'une éphémère fondation monastique effectuée aux portes de Lisieux sous l'épiscopat de Fréculf}

The Tale of the Translation of Saint Regnobert' Relics: History of a transitory monastic foundation created on the Outskirts of Lisieux under Freculfus' episcopate

\section{Christophe Maneuvrier}

\section{OpenEdition}

\section{Journals}

Édition électronique

URL : http://journals.openedition.org/tabularia/1437

DOI : $10.4000 /$ tabularia. 1437

ISSN : 1630-7364

Éditeur :

CRAHAM - Centre Michel de Boüard, Presses universitaires de Caen

Référence électronique

Christophe Maneuvrier, «Le récit de la translation des reliques de saint Regnobert : histoire d'une éphémère fondation monastique effectuée aux portes de Lisieux sous l'épiscopat de Fréculf », Tabularia [En ligne], Écrire l'histoire au Moyen Âge, mis en ligne le 04 janvier 2005, consulté le 30 avril 2019. URL : http://journals.openedition.org/tabularia/1437 ; DOI : 10.4000/tabularia.1437 


\title{
Le récit de la translation des reliques de saint Regnobert: histoire d'une éphémère fondation monastique effectuée aux portes de Lisieux sous l'épiscopat de Fréculf
}

\section{The Tale of the Translation of Saint Regnobert' Relics: History of a transitory monastic foundation created on the Outskirts of Lisieux under Freculfus' episcopate}

\author{
Christophe MANEUVRIER \\ CRAHM-UMR 6577 \\ Université de Caen Basse-Normandie \\ ch.maneuvrier@wanadoo.fr
}

Résumé:

Bien que publié à plusieurs reprises depuis le XVII ${ }^{e}$ siècle, le récit de la translation des reliques de saint Regnobert demeure mal connu. L'identification des noms de lieux et des personnages permet de proposer ici une nouvelle lecture de ce texte qui, rédigé en deux temps, d'abord vers $856 / 858$ puis vers $867 / 869$, relate le périple suivi par les reliques depuis Bayeux jusqu'aux portes de la cité épiscopale de Lisieux. C'est en effet à Suiacum, sur une terre qui relevait alors de la juridiction de l'évêque de Bayeux, que fut fondé vers le milieu du IX ${ }^{\mathrm{e}}$ siècle un petit établissement monastique destiné à accueillir les reliques du saint. Durant sa courte existence, ce monastère reçut plusieurs aumônes dont quelques-unes de Charles le Chauve et de la reine Ermentrude.

Mots-clés : Translation, reliques, saint Regnobert, fondation monastique, Charles le Chauve, Ermentrude, Lisieux, Bayeux, Hervé, Suiacum.

\section{Abstract:}

Although it has been published several times since the $17^{\text {th }}$ century, the tale of the translation of saint Regnobert's relics remains little known. The identification of place-names and characters makes it possible to propose here a new reading of this text. The latter, written in two stages, first around 856/858 A.D., then around 867/869 A.D., tells of the journey of the relics from Bayeux to the outskirts of the episcopal city of Lisieux. It is indeed at Suiacum, on a land that fell at the time under the juridiction of the bishop of Bayeux, that a little monastic settlement was created around the middle of the $9^{\text {th }}$ century, in order to shelter the saint's relics. Over the course of its short existence, this monastery received several alms, among which some from Charles the Bald and Queen Ermentrude.

Keywords: Translation, relics, Regnobert (saint), monastic foundation, Charles the Bald, Ermentrude, Lisieux, Bayeux, Suiacum. 
L'Historia translationis corporum sanctorum Ragnoberti et Zenonis bénéficie, depuis le XVII siècle, d'une image exécrable, l'historicité de la translation de ses reliques ayant même été mise en doute par les Bollandistes, puis par le clergé bayeusain luimême ${ }^{1}$. Le texte de ce récit fut publié pour la première fois en 1675 par Dom Luc d'Achery d'après un manuscrit conservé dans l'abbaye de Saint-Bénigne de Dijon. Quelques années plus tard, les Bollandistes en livrèrent une autre version établie d'après des manuscrits découverts à Belfort, Vivant-sous-Vergy et Auxerre ${ }^{2}$. Un troisième texte, légèrement différent, fut publié peu après dans la Neustra Pia, à la suite de la vita de saint Regnobert, d'après un manuscrit provenant cette fois de la collégiale de $\mathrm{Varzy}^{3}$. Enfin, l'ensemble de ces versions fut compilé et publié en 1952 par l'abbé Larue, accompagné de notes et de commentaires féroces, affirmant par exemple que «tout y est fantaisie ${ }^{4}$. Victime de cet anathème positiviste, l'Historia fut longtemps laissée de côté, tant par les historiens de la Normandie que par les spécialistes d'histoire religieuse ${ }^{5}$.

Une autre tradition historiographique reconnaît pourtant au récit une certaine valeur historique ${ }^{6}$. Dès 1835, l'érudit normand Auguste Le Prévost proposait plusieurs identifications de noms de lieux et corrigeait certaines attributions erronées faites, avant lui, par les Bollandistes ${ }^{7}$. Quelques années plus tard, Jules Lair affirma que le récit de la translation n'était pas aussi mauvais que le disaient les Bollandistes, sa datation pouvant être située dans le IX e siècle, sans doute avant $877^{8}$. En 1904, Léon Levillain découvrit même l'identité de l'auteur de l'Historia translationis corporum sanctorum Ragnoberti et Zenonis grâce à un manuscrit resté inconnu des Bollandistes ${ }^{9}$. Il s'agit d'un prêtre nommé Joseph, que Jules Lair et Léon Levillain ont proposé d'identifier avec l'un des notaires de la chancellerie du roi Pépin II d'Aquitaine attesté par trois diplômes datés de janvier 846, de mai 847 et de février $848^{10}$, Joseph se qualifiant lui-même, dans le chapitre XVII de l'Historia, de «prêtre, notaire du feu roi d'Aquitaine, maintenant précepteur et chancelier du roi Louis». L'Historia nous apprend également que, passé au service de Charles le Chauve, Joseph devint en 856 le précepteur et le chancelier de Louis le Bègue et qu'auparavant il fut l'élève d'Amauri, à Tours, où ils eurent pour condisciple Paulus, archevêque de Rouen. Quel

1. LAIR, 1863, p. 102.

2. Acta Sanctorum, 17 Mai, 1680, tome III, p. 615-624.

3. Tous ces manuscrits semblent avoir aujourd'hui disparu. Seule subsiste à la Bibliothèque nationale une copie du manuscrit de Varzy faite par Du Monstier de la vita publiée par Jules Lair (LAIR, 1863).

4. LARUE, 1952.

5. GEARY, 1993; AlLEN, 1994.

6. Le souvenir de cette translation figure déjà dans l'ordinaire du XIII ${ }^{\mathrm{e}}$ siècle. Ordinaire et coutumier de l'église cathédrale de Bayeux, éd. Ulysse Chevalier, Paris, 1902, p. 245.

7. LE PrÉvost, 1835.

8. LAIR, 1863 , p. 106

9. LeVillain, 1904, p. 295.

10. Janet Nelson signale que Joseph fut également chanoine de Bayeux, mais, comme l'a fort justement souligné Michael Allen, la source de cette affirmation demeure inconnue. NELSON, 1994; ALLEN, 1994. 
rôle Joseph joua-t-il dans cette affaire? Sans doute n'était-il pas en Neustrie lors de la translation puisqu'il se trouvait à ce moment là au service de Pépin II $^{11}$. Le texte comprend pourtant deux verbes à la première personne du pluriel (in sui translatione [corporis] vidimus) mais il se pourrait que l'auteur reprenne ici une source écrite plus ancienne. Le texte est, en tous cas, postérieur à l'année 864, date de la disparition de Pépin II et probablement même à 867, année durant laquelle Charles le Chauve donna l'Aquitaine à son fils Louis. Remarquant que Charles le Chauve n'est qualifié dans le récit que de rex et soulignant le fait que l'Historia fut rédigée lorsque la reine Ermentrude était encore en vie, Léon Levillain est finalement arrivé à dater ce récit des années $867 / 869^{12}$.

L'Historia précise cependant que ce récit fut écrit à la demande de Paul, archevêque de Rouen, mort vers 858 . Force est donc de constater, comme le fit Léon Levillain, que ce texte fut écrit en deux temps : commencé sous l'archiépiscopat de Paul (mort en 858) et alors que l'évêque de Bayeux Baltfridus semble être encore en vie (massacré par les Normands en 858 ), il fut terminé ou mis au propre une douzaine d'années plus tard, entre 867 et 869 . En raison de la mention de l'entrevue de Veteres Domus entre Charles le Chauve et le breton Erispoé, on peut dater la première phase de rédaction de l'Historia des années 856-858, c'est-à-dire durant la période au cours de laquelle la Neustrie fut déchirée par une violente crise politique. En 856, Louis le Bègue fut investi du regnum sur la Neustrie et Joseph (l'auteur de l'Historia) est désigné comme son précepteur ou tuteur ${ }^{13}$, mais en 858 , Erispoé - le protecteur de Louis le Bègue - est renversé par Salomon. Robert le Fort, avec l'aide de ses alliés Bretons, en profite pour chasser Louis le Bègue et sa suite de Neustrie ${ }^{14}$. C'est alors en Bourgogne, autour d'Auxerre, que Charles le Chauve refait ses forces. Or, c'est justement dans la région d'Auxerre que les reliques de saint Regnobert trouvèrent refuge en suivant une route qui les conduisit, d'après Lucien Musset, d'abord à SaintVivant-sous-Vergy ${ }^{15}$, puis à Varzy ${ }^{16}$, Quingey ${ }^{17}$.

La première phase de rédaction, celle des années 856-858, faite à la demande de Paul, a pour objet de rapporter l'histoire de ces reliques sans doute au moment où elles quittent la province ecclésiastique de Rouen pour la Bourgogne. La seconde phase de rédaction doit correspondre à une réécriture ou à une mise au net probablement effectuée autour d'Auxerre, vers 867-869.

11. LAIR, 1863 , p. 115

12. LeVILlain, 1904.

13. Nelson, 1994, p. 205.

14. NELSON, 1994, p. 208.

15. Dép. Côte-d'Or, cant. Gevrey-Chambertin, commune de Curtil-Vergy.

16. Dép. Nièvre, chef-lieu de cant.

17. Dép. Doubs, chef-lieu de cant. Quelques reliques furent peut-être également expédiées à Corbeil (dép. Essonne, chef-lieu de cant.; MUSSET, 2000, p. 102). 


\section{La première translation des reliques de Bayeux à Saint-Victor}

La première partie du récit est tout à fait classique. Le responsable de la translation - ou du vol des reliques si on suit la classification de Patrick Geary - s'entoure de toutes les autorisations nécessaires: celles des saints Regnobert et Zénon, d'abord, qui, par trois fois, lui donnent l'ordre d'aller à Bayeux chercher leurs reliques menacées par la présence des Bretons dans les parages; celle de Fréculf ensuite, l'évêque de Lisieux. Lui manque celle de Baltfridus, évêque de Bayeux, qu'il recherche en vain mais qu'il n'arrive pas à rencontrer, l'évêque étant alors en train de se justifier, à l'extérieur de son diocèse, d'une attaque dont on ignore la nature.

L'auteur insiste ensuite sur le fait qu'Hervé, le responsable de la translation, est un bon chrétien: il part pour Bayeux le jour de Noël 846 après avoir assisté aux offices, accompagné pour l’occasion par deux prêtres désignés par Fréculf. Arrivé dans l'église Saint-Exupère de Bayeux, où sont conservées les précieuses reliques, il prend le temps de prier et de se recueillir. Bon chrétien, il ne peut en effet se soustraire à la mission dont l'ont chargé les saints qui est de mettre leurs reliques à l'abri.

Les reliques qu'Hervé va chercher à Bayeux sont celles de saint Regnobert, évêque de Bayeux au VII ${ }^{e}$ siècle ${ }^{18}$, pour lequel on possède une vita du VIII ${ }^{e}$ ou du IX ${ }^{e}$ siècle ${ }^{19}$, et de saint Zénon, simplement qualifié de diacre et sur lequel on ne sait rien. Qui est cet Hervé? L'Historia précise qu'il est originaire du Maine. Peut-être est-ce le même personnage que celui qui, nommé Heireveus, figure avec plusieurs comtes parmi les témoins d'un acte de Charles le Chauve pour l'église de Rouen daté du 4 novembre $863^{20}$. Son nom et son origine géographique nous incitent à le rapprocher des Rorgonides. Sans doute est-il également apparenté à la famille des comtes du Perche qui, au IX et $\mathrm{X}^{\mathrm{e}}$ siècle, portaient fréquemment le nom d'Hervé ${ }^{21}$. Cherche-t-il vraiment à mettre ces reliques à l'abri ou bien veut-il simplement profiter de l'absence de l'évêque Baltfridus pour enrichir un sanctuaire familial?

L'étude de l'itinéraire des reliques permet de mettre un peu en lumière l'assise territoriale de la puissance d'Hervé. Ainsi, après avoir quitté Bayeux, Hervé fait une

18. SIMON, 1947.

19. LAIR, 1862.

20. TESSIER, $1943, \mathrm{n}^{\circ} 259$, p. 88-89.

21. Une branche des Rorgonides, représentée par les comtes d'Herbauge, fut placée sur la marche de Bretagne où elle était chargée de défendre la Neustrie contre les Normands et les Bretons, le propre fils de Renaud d'Herbauge, portant le nom d'Hervé. En 912, Un certain Herveus, comes, souscrit un acte pour l'abbaye de Marmoutiers puis, en 955, un autre Herveus est gratifié cette fois du titre de « comte de Mortagne ». La présence de cette même famille en Lieuvin et dans le Perche est probablement à l'origine des liens entretenus du IX ${ }^{\mathrm{e}}$ au XII ${ }^{\mathrm{e}}$ siècle par l'abbaye de Corbion/Saint-Laumerde-Blois avec quelques localités des environs de Lisieux. Sur ces aspects, voir nos développements dans MANEUVRIER, 2000. 
première étape in villa quae vocatur Nogerolas ${ }^{22}$, localité identifiée depuis les Bollandistes et Auguste Le Prévost, avec celle de Norolles ${ }^{23}$. Cette proposition est parfaitement recevable si l'on considère que les communes actuelles de Norolles et de Fauguernon ${ }^{24}$ ne constituaient alors qu'une seule villa. Le passage des reliques de saint Regnobert donna lieu à la construction, sur le territoire de la commune actuelle de Fauguernon, d'un petit sanctuaire dédié à saint Regnobert qui, encore qualifié de capella en 1221, finit par acquérir tardivement l'autonomie paroissiale ${ }^{25}$. Le texte de la translation ne précise pas les raisons pour lesquelles Hervé choisit de passer la nuit à Norolles/Fauguernon. Hervé y possédait peut-être une résidence sur l'éperon rocheux qui domine l'église Saint-Regnobert, à l'emplacement où, plus tard, sera édifié l'un des plus puissants châteaux de la famille Bertran ${ }^{26}$.

De Norolles, les reliques furent ensuite transportées vers une basilique SaintVictor, identifiée par Auguste Le Prévost avec l'église de Saint-Victor-d'Epine ${ }^{27}$. Le texte de la translation précise qu'Hervé tenait cette terre de sa mère, ce qui explique son éloignement du Maine, berceau de la famille paternelle d'Hervé. Cependant, le choix de la dédicace de cette église révèle peut-être une influence mancelle puisque le saint honoré ici est en réalité saint Victeur, évêque du Mans à la fin du $\mathrm{V}^{\mathrm{e}}$ siècle $^{28}$. Les relations entre Hervé et le Maine expliquent également la présence, parmi les malades guéris miraculeusement dans la basilique Saint-Victor, d'un aveugle originaire de la cité du Mans ${ }^{29}$.

C'est probablement le retour de Baltfridus à Bayeux qui obligea Hervé à composer avec les autorités religieuses bayeusaines, l'amenant à accepter une restitution partielle des reliques en les confiant à un centre monastique qu'il créa pour l'occasion sur ses terres en un lieu relevant de la juridiction de l'évêque de Bayeux. Le texte, un peu flou à ce sujet, témoigne de la gène de l'auteur qui se contente de rapporter les dires d'une femme selon laquelle saint Regnobert lui serait apparu et lui aurait

22. Interea pergentes praefati viri cum sanctorum corporibus, vespere facto divertunt ad quietem in villa quae vocatur Nogerolas et ibidem nocte illa quiescentes in divinis officiis noctem pervigilem duxerunt, chap. III.

23. Dép. Calvados, cant. de Blangy-le-Château.

24. Dép. Calvados, cant. de Lisieux.

25. Confirmation des biens du prieuré de Beaumont-en-Auge par Robert Bertran de 1221 : et sciendum est quod capelle sancti Nicholai de Ronchevilla et sancti Renoberti de Fausguernon, michi remanent. BRÉARD, 1908, n 19, p. 205-207.

26. Mal documenté, le château de Fauguernon est mentionné pour la première fois en 1137 (CAUMONT, p. 34). Quelques tessons de céramique gallo-romaine (commune, sigillée et métallescente) et des fragments de tegulae ont été découverts dans l'enceinte du château de Fauguernon. Information inédite communiquée par M. Gurrey, propriétaire actuel du château.

27. Dép. Eure, cant. Brionne. On pourrait également proposer Saint-Victor-de-Chrétienville (dép. Eure, cant. Bernay), mais la route la plus directe en provenance de Bayeux passe par Lisieux alors que celle qui conduit de Bayeux à Saint-Victor-d'Epine passe par Norolles et Fauguernon.

28. Ce culte est formellement attesté à Saint-Victor-de-Chrétienville. ForMEVILLE, 1872, vol. I, p. cii ; CHARLES, 1980, p. 32-33; BIARNE, 1987, p. 48.

29. LARUE, 1952, p. 227. 
annoncé qu'il ne ferait plus de miracle tant que son corps n'aurait pas été déplacé dans un lieu pur, non souillé par les âmes des damnés.

\section{La $2^{\mathrm{e}}$ translation des reliques de Saint-Victor à Suiacum}

De Saint-Victor, les reliques furent transférées par Hervé dans une église nouvellement construite, localisée dans une villa appelée Suiacum. L'identification de ce lieu n'a jusqu'à présent jamais été résolue de façon satisfaisante. Ferdinand Lot, reprenant une proposition de Jules Lair, identifiait Suiacum avec Notre-Dame-d'Epine, localité voisine de Saint-Victor-d'Epine ${ }^{30}$. Toutefois, l'église construite par Hervé à Suiacum était selon l'Historia dédiée au Sauveur ${ }^{31}$. Il est donc tentant d'identifier cette localité avec le fief de Suilly, sur la commune actuelle de Manerbe, où il existait une chapelle Saint-Sauveur encore qualifiée d'église dans le pouillé du XIVe siècle (ecclesia seu capella sancti Salvatoris) ${ }^{32}$. Vendue comme bien national le 16 juin 1791, cette chapelle fut détruite peu après et ses matériaux réutilisés pour la construction d'une maison ${ }^{33}$. Toutefois, son emplacement est encore signalé par le cadastre napoléonien (fig. 1). Le souvenir de la courte installation des moines de Suiacum y est également conservé sous la forme d'un microtoponyme; le chemin passant devant la chapelle portant le nom des «chemin aux Moines».

Les raisons du choix d'Hervé sont en partie expliquées par l'auteur de l'Historia: selon Joseph, les saints Regnobert et Zénon auraient ordonné à Hervé de construire une église «en un lieu pur et non souillé, les saints ne supportant pas que leurs corps soient placés auprès d'autres corps dont les âmes seraient en enfer ${ }^{34}$. Cette église Saint-Sauveur fut donc établie dans une zone boisée - peut-être défrichée pour l'occasion à moins qu'il ne s'agisse que d'un lieu commun de l'hagiographie carolingienne -, sur le bord de l'ancienne route qui conduisait de Lisieux à Dives-surMer. Mais surtout, la terre de Suilly relevait de l'exemption de Cambremer qui dépendait, depuis le $\mathrm{VI}^{\mathrm{e}}$ siècle, de l'évêché de Bayeux ${ }^{35}$, ce qui explique pourquoi

30. LOT, 1904, p. 468 ; LAIR, 1863, p. 115, note 2.

31. C'est sur cette base que Henri Pellerin a proposé de placer Suiacum à Beaumont-en-Auge où l'on trouve la seule église du diocèse de Lisieux placée sous cette dédicace. Cette proposition peut paraître séduisante, d'autant que l'église de Beaumont-en-Auge est peu éloignée de Norolles et qu'elle était le centre d'un prieuré fondé vers 1050 par la famille Bertran. Cette hypothèse nous paraît cependant beaucoup moins plausible que celle qui est proposée ici. PELLERIN, 1957.

32. LONGNON, 1903, p. 128. Manerbe: dép. Calvados, cant. Blangy-le-Château. Le fief de Suilly, qui relevait de l'honneur de Manerbe, est attesté pour la première fois en 1259. (Caen, Arch. dép. Calvados, F 4845, chartrier du Pré-d'Auge). En 1530, «la terre et sieurie du Pré d'Auge anciennement appelée et nommée de Silly» compte pour un quart de fief noble relevant de la seigneurie de Manerbe. Arch. dép. Calvados, F 4845 (chartrier du Pré-d’Auge).

33. Cette maison n'a malheureusement pas pu être localisée. "Histoire manuscrite de Manerbe», Arch. de la Société historique de Lisieux, fonds Deville. Je remercie Jean-Alain Cairon qui m’a signalé l'existence de ces documents.

34. LARUE, 1952, p. 225-227 et 248 .

35. Sur l'histoire de cette exemption depuis le VI ${ }^{\mathrm{e}}$ siècle, voir Arnoux et Maneuvrier, 2000. 


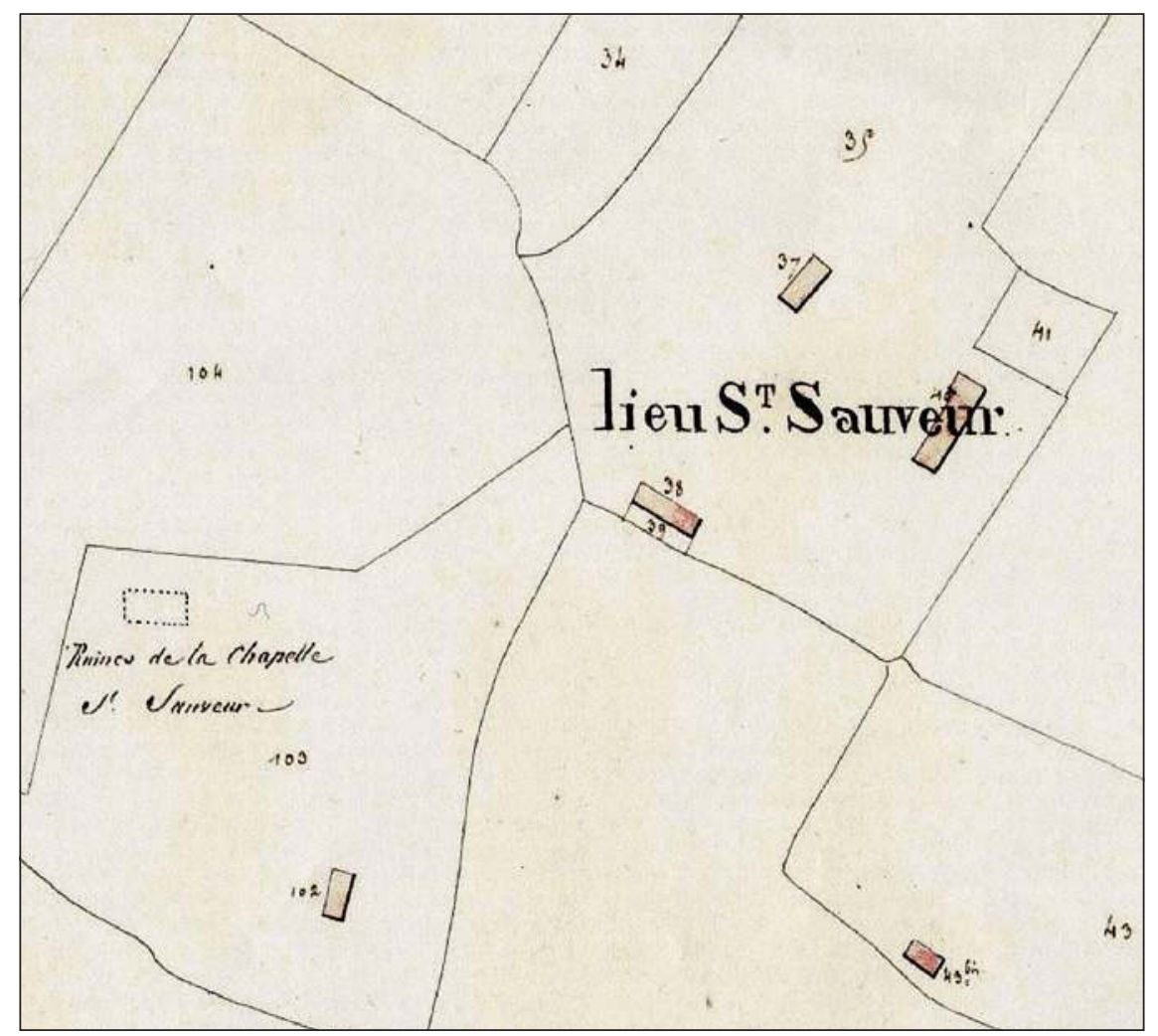

Fig. 1 : Extrait du cadastre napoléonien (sans date, vers 1825) indiquant l'emplacement de la chapelle Saint-Sauveur de Suilly. (Arch. dép. Calvados, 3P 5060, parcelle B 101)

le texte de l'Historia indique que Baltfridus, évêque de Bayeux, vint assister à la cérémonie accompagné d'une foule immense. En installant les reliques de saint Regnobert et de saint Zénon à Suilly, Hervé les rendait en partie à l'évêque de Bayeux tout en les conservant sur ses terres, à quelques kilomètres de la cité lexovienne. Si la première translation des reliques, de Bayeux à Saint-Victor, s'apparente à un vol, la seconde translation qui conduisit les reliques de Saint-Victor à Suiacum offre l'apparence d'un retour vers l'évêché de Bayeux. On s'explique mieux ainsi le faste de la dédicace de cette église effectuée le 25 mars 847, 848 ou 849, en présence de Fréculf, évêque de Lisieux, de Baltfridus évêque de Bayeux et d'Ansegaudis, évêque d'Avranches. Si la présence de Fréculf et de Baltfridus s'expliquent aisément - le premier est présent parce que cette fondation est réalisée aux portes de sa cité, le second parce qu'il est le successeur de Regnobert et parce que la terre de Suilly est située sous sa juridiction épiscopale - on s'explique moins la présence d'Ansegaudis. Peut-être faut-il se demander si la cité de Lisieux n'aurait pas servi, vers le milieu du IX siècle, de base de repli pour les prélats de la province ecclésiastique de Rouen les plus exposés aux menaces bretonnes. 
L'aspect extérieur de l'édifice demeure inconnu, le rectangle mentionné sur le cadastre napoléonien n'étant sûrement qu'un signe conventionnel. L'Historia précise toutefois que l'église comprenait trois autels « in qua tres Deo omnipotenti arae constructae erant» et qu'au centre de l'édifice furent placés les corps de saint Regnobert et de saint Zénon. Fréculf, évêque de Lisieux, consacra le premier autel dédié au saint Sauveur; Baltfridus, évêque de Bayeux, celui du «milieu » dédié à saint Regnobert et Ansegaudis, évêque d'Avranches, celui dédié à la Vierge et à saint Jean-Baptiste ${ }^{36}$.

Le rayonnement de la petite communauté monastique installée à Suiacum semble avoir été surtout local, les miracles rapportés par l'Historia concernant presque toujours des personnages des environs immédiats de Suilly. Plusieurs sont originaires de Babiola villa, localité identifiée jusqu'à présent avec Bailleul-la-Vallée ${ }^{37}$ mais qu'il semble préférable de placer à Bailleul, simple hameau de la commune voisine de Coquainvilliers ${ }^{38}$. Le texte mentionne également un voleur de gerbes, puni de mort par les saints, originaire de villam Promerendum nomine ${ }^{39}$, localité qui doit être celle de La Pommeraye sur la commune actuelle de Saint-Désir ${ }^{40}$. Toutefois, la communauté de Suiacum bénéficia, vers 856, de plusieurs aumônes royales. Charles le Chauve, de passage à Veteres Domus (Louviers?), souffrant d'un mal de dents, envoya son «vœu» au tombeau des saints tandis que la reine Ermentrude, atteinte d'un mal de poitrine, y fit porter une pièce d'étoffe pour couvrir les tombeaux des saints (pallium ad Beatorum corpora). Elle persuada également Charles le Chauve de donner quelques terres à cette église ${ }^{41}$. Le déménagement effectué peu après vers la Bourgogne empêcha cependant le développement de cette petite communauté monastique.

L'Historia translationis corporum sanctorum Ragnoberti et Zenonis appartient bien à un genre littéraire stéréotypé de la seconde moitié du IX ${ }^{\mathrm{e}}$ siècle, et elle contient de nombreux lieux communs que l'on rencontre dans d'autres récits du même type. Toutefois, ce texte, œuvre d'un grand lettré, répond à la volonté de produire une

36. «Etenim tria ibidem altaria trium praesulum consecrationibus sanctificantur quorum primum in honore Domini nostri Jesu Christi venerabilis Freculfus Lixogensis episcopus consecravit; medium Baltfridus antistes Baiocensis ecclesiae, in honore sancti Regnoberti et omnium sanctorum cum Zenonis sanctificavit, quod ad capita confessorum videbatur esse fundatum; tertium enim pontifex Abrincatensis urbis Ansegaudus in veneratione sanctae genitricis Mariae et sancti Joannis Baptistae benedixit». Chap. 7, éd. Larue, 1852 , p. 230.

37. Dép. Eure, cant. Cormeilles. Babiolas, Babiola villa ou Baliola villa. DAUZAT et RostAING, 1978, p. 47. LARUE, 1952, p. 233-234.

38. Dép. Calvados, cant. Blangy-le-Château. Ce fief disposait d'un moulin attesté par un microtoponyme conservé par le cadastre dit napoléonien: "Moulin de Bailleul », commune de Coquainvilliers, Arch. dép. Calvados, 3P 5058 parcelles B. 56-62. Sur le terrain, on discerne encore très nettement les restes d'un ancien bief.

39. LaRUe, 1952, p. 235-237.

40. Dép. Calvados, cant. Lisieux. Une chapelle succursale de l'église Saint-Désir-de-Lisieux dédiée à saint Laurent y est signalée au début du XIV e siècle (Caen, Arch. dép. Calvados, H non côté «Saint-Désir» 140, carton 1), mais la construction actuelle remonte à l'époque romane. CAUMONT, 1867, p. 306-308.

41. Chapitre XVIII-Larue, p. 238-239. 
histoire, celle d'une éphémère fondation monastique effectuée aux portes de la cité lexovienne. Transporté en Auxerrois en même temps que les reliques (vers 856-858), ce récit fut rapidement oublié dans la province ecclésiastique de Rouen, Suiacum/ Suilly n'étant plus rapidement qu'un nom perdu, vide de sens.

L'identification des personnages et des lieux du récit montre toutefois que le récit de cette translation ne peut plus être rejeté en bloc comme l'ont fait si longtemps les héritiers de l'histoire positiviste. Sans doute serait-il souhaitable de disposer pour ce texte d'une nouvelle édition critique, qui permettrait en particulier de travailler sur la langue et la culture de l'auteur. Une première campagne de sondages archéologiques, prévue pour l'été 2004 sur le site de la chapelle Saint-Sauveur de Suilly, permettra peut-être également de mieux connaitre l'organisation de cet établissement monastique méconnu.

\section{Bibliographie}

ACHERY, Dom Luc d', (éd.), Spicilegium sive collectio veterum aliquot scriptorum qui in Galliae bibliothecis delituerant, Paris, 1675, t. XII, p. 611-621.

Acta Sanctorum, 17 Mai, 1680, « Historia translationis corporum sanctorum Ragnoberti et Zenonis », tome III, p. 615-624.

ALLEN, Michael Idomir, History in the carolingian renewal: Frechulf of Lisieux (FL. 830), His Work and Influence, Centre for Medieval, Studies university of Toronto, Thesis submitted for the degree of Doctor of Philosophy, june 1994, 376 p. (dactyl.).

ARnoux, Mathieu et MANEUVRIER, Christophe, Deux abbayes de Basse-Normandie: Notre-Dame-du-Val et le Val-Richer (XII ${ }^{-}$-XIII ${ }^{e}$ siècles), Flers, 2001, Le Pays Bas Normand, n²37-238, $111 \mathrm{p}$.

BAUDUIN, Pierre, La frontière normande aux $X^{e}-X I^{e}$ siècles. Origine et maîtrise de la frontière sur les confins de la Haute Normandie (911-1087), Thèse de doctorat, Université de Caen, UFR d'Histoire, 1998, 2 vol., 562 p. (dactyl.).

BIARNE, Jacques, «Le Mans», Topographie chrétienne des cités de la Gaule, des origines au milieu du VIII siècle. Tome V: Province ecclésiastique de Tours, Paris, 1987, p. 41-56.

BOUVRIS, Jean-Michel, «Les plus anciennes chartes du prieuré de Saint-Sulpice près de L'Aigle, dépendance normande de l'abbaye de Saint-Laumer de Blois, $\mathrm{XI}^{\mathrm{e}}-\mathrm{XII}{ }^{ }$», Annales de Normandie, 30, nº 4, décembre 1981, p. 327-330.

BRÉARD, Charles, Cartulaires de Saint-Ymer-en-Auge et Bricquebec, Rouen, Lestringant et Paris, Picard, 1908, XLV-343 p.

BRUNTERC'H, Jean-Pierre, "Le duché du Maine et la marche de Bretagne », La Neustrie. Les pays au nord de la Loire de 650 à 850 . Colloque historique international publié par Hartmut Atsma, Beihefte der Francia, herausgegeben 
vom Deutschen Historischen Institut, Paris, Bd. 16.2, Sigmaringen, 1989, tome 1, p. 29-127.

CAUMONT, Arcisse de, Statistique monumentale du Calvados, tome V, arrondissement de Lisieux, Caen-Paris, 1867, réimpression 1978, Mayenne, Joseph Floch, $847 \mathrm{p}$.

DAUZAT, Albert, et Rostaing, Charles, Dictionnaire étymologique des noms de lieux en France, Paris, Larousse, 1963, réimpression 1978, XII-738 p.

Formeville, Henri de, Histoire de l'ancien évêché-comté de Lisieux, 2 vol., 1872 (réimpression, Brionne, le Portulan, 1971), DCLIII-419 p.

GAZEAU, Véronique, «Le patrimoine d'Hugues de Bayeux (c. 1011-1049)», Les évêques normands du XI ${ }^{e}$ siècle, François NeveuX et Pierre Bouet (éd.), Colloque de Cerisy-la-Salle, Caen, Presses universitaires de Caen, 1995, p. 139-147.

GEARY, Patrick G., Le vol des reliques au Moyen Âge, Paris, Aubier, 1993, Histoire, $256 \mathrm{p}$.

Guillotel, Hubert, «Le temps des rois, $\mathrm{IX}^{\mathrm{e}}-\mathrm{X}^{\mathrm{e}}$ siècles », André CHÈDEVILLE et Hubert Guillotel (éd.), La Bretagne des saints et des rois (Ve-Xe siècles), Rennes, Ouest-France/Université, 1984, 423 p.

LAIR, Jules, «Etudes sur les origines de l'évêché de Bayeux", Bibliothèque de l'École des Chartes, tome III, année 1862, p. 89-124 et tome IV, année 1868, p. 52-546.

LARUE, O. (abbé), «La translation des corps de saint Regnobert et de saint Zénon (Texte et critique) ", Bull. de la Soc. des Antiquaires de Normandie, tome LI, années 1948-51, 1952, p. 217-264.

LAUER, Philippe et LOT, Ferdinand, Recueil des actes de Charles III le Simple (893923), Paris, Académie des Inscriptions et Belles-Lettres (Chartes et diplômes relatifs à l'histoire de France), 1940-1949, 2 vol.

Tessier, Georges, Recueil des actes de Charles II le Chauve, roi de France, 3 vol., Paris, Académie des Inscriptions et Belles-Lettres (Chartes et diplômes relatifs à l'histoire de France, 9), 1943-1955.

Le PrÉvost, Auguste, « Notes pour servir à l'histoire de Normandie», Annuaire des cinq départements de l'ancienne Normandie, tome I, 1835, p. 25-26.

LEVILLAIN, Léon, «La translation des reliques de saint Austremoine à Mozac et le diplôme de Pépin II d'Aquitaine (863) », Le Moyen Âge, 2e série, tome VIII, juillet-août 1904, p. 281-337.

LONGnON, Auguste, Pouillés de la province de Rouen, Paris, Klincksieck, 1903, LXXV-602 p.

LOT, Ferdinand, "Mélanges carolingiens I. Veteres domus», Le Moyen Âge, tome XVII, novembre-décembre 1904, p. 465-477.

LOUISE, Gérard, La seigneurie de Bellême (Xe-XIIe siècles). Dévolution des pouvoirs territoriaux et construction d'une seigneurie de frontière aux confins de la Normandie et du Maine à la charnière de l'an Mil, Flers, Le Pays Bas-Normand, 2 vol., 1992, $432+349$ p. 
MANeuvrier, Christophe, Paysages et sociétés rurales au Moyen Âge. Le Pays d'Auge jusqu'à la fin du XIII' siècle, Thèse de doctorat, Caen, UFR d'Histoire, 19992000, 3 vol. 571 p. + 111 fig. et 15 tabl. +79 p. (dactyl.).

MUSSET, Lucien, «L'exode des reliques du diocèse de Sées au temps des invasions normandes", Bulletin de la Société historique et archéologique de l'Orne, tome LXXXVIII, 1970, p. 3-22.

Musset, Lucien, "Autour de saint Lhomer et de Corbion », Annuaire de l'Association Normande, tome 152, Congrès d'Alençon (1994), 1995, p. 71-74.

MUSSET, Lucien, «Les translations de reliques en Normandie», Les Saints dans la Normandie médiévale, Colloque de Cerisy-la-Salle (26-29 septembre 1996), Pierre BouET et François NEveux (éd.), Caen, Presses universitaires de Caen, 2000, p. 97-108.

Nelson, Janet, L., Charles le Chauve, Paris, Aubier, 1994, 404 p.

PelLerin, Henri, «La translation des reliques de saint Regnobert en Pays d'Auge et les origines religieuses de Beaumont-en-Auge », Le Pays d'Auge, n 12, décembre 1957, p. 4-11.

RAMACKERS, Johannes, Papsturkunden in Frankreich. 6. Band Orléanais, Göttingen, 1958, (Abhandlungen der Gesellschaft der Wissenschaften zu Göttingen, phil.-hist. Klasse, 3. Folge 41).

Simon, Georges-Abel, «Les origines diocésaines de Bayeux et les anciennes listes épiscopales ", Cahiers Léopold Delisle, tome I, fasc 1, Paris, 1947, p. 57-74.

Tessier, Georges, Recueil des actes de Charles II le Chauve, roi de France, 3 vol., Paris, Académie des Inscriptions et Belles-Lettres (Chartes et diplômes relatifs à l'histoire de France, 9), 1943-1955. 